\title{
Variation in colorectal cancer testing between primary care physicians: \\ A cross-sectional study in Switzerland
}

\begin{abstract}
Alexander Leonhard Braun ${ }^{1}$, Emanuele Prati $^{1}$, Yonas Martin ${ }^{1}$, Charles Dvořák ${ }^{2}$, Kali Tal ${ }^{1}$, Nikola Biller-Andorno ${ }^{3}$, Jean-Luc Bulliard ${ }^{4}$, Jacques Cornuz ${ }^{5}$, Kevin Selby, ${ }^{5,6}$ Reto Auer ${ }^{1,5}$
\end{abstract}

\footnotetext{
${ }^{1}$ Institute of primary health care (BIHAM), University of Bern, Switzerland, ${ }^{2}$ President Sentinella Network, Switzerland, ${ }^{3}$ Institute for Biomedical Ethics and History of Medicine (IBME), Zürich, Switzerland, ${ }^{4}$ Institute of Social and Preventive Medicine (IUMSP), Lausanne University Hospital (CHUV), Switzerland, ${ }^{5}$ Department of Ambulatory Care and Community Medicine, University of Lausanne, Switzerland, ${ }^{6}$ Northern California Division of Research, Kaiser Permanente, United
} States of America 
Word count: Abstract : 180 words, Text body: 3437 Words

Key Words: primary care, colorectal cancer, screening, practice variation, decision making

\section{Abstract}

Objective: To determine the proportion of 50-75-year-old patients of primary care physicians (PCP) who were tested for colorectal cancer (CRC) by either colonoscopy within 10 years or fecal occult blood testing (FOBT) within 2 years. To describe the variation in care between PCPs and factors associated with these proportions.

Methods: Cross-sectional study between April and December 2017. Participants: PCPs reporting for the Swiss Sentinel Surveillance Network. Each PCP collected demographic data and CRC testing status from 40 consecutive patients. Measurements: proportions of patients up-to-date with CRC screening and method used (colonoscopy/FOBT/Other); variation in the outcome measures between PCPs; association of physician-level factors with main outcomes.

Results: 91/129 PCPs collected data from 3,451 patients; 45\% had been tested for CRC within recommended intervals (41\% colonoscopy, 4\% FOBT). The proportions of patients tested and testing with colonoscopy vs. FOBT varied widely between PCPS. Language region was associated with PCPs' rate of FOBT prescription.

Conclusion: Less than half of patients who visited PCPs in Switzerland were up-to-date with CRC screening recommendations. PCPs varied widely in their testing practices. 


\section{Introduction}

Screening for colorectal cancer (CRC) is recommended for those over 50 at average-risk of CRC, based on evidence from randomized controlled trials and observational studies that CRC screening substantially reduces CRC mortality and decreases incidence. (Brenner et al. 2014; Dominic et al. 2009; Lauby-Secretan et al. 2018; USPSTF 2016; von Karsa et al. 2013) Fewer people in Europe are screened for CRC than in the US. (Chen et al. 2017; Klabunde et al. 2015; Stock and Brenner 2010) In Switzerland, the 2012 national health survey found that only $40 \%$ of 50-75 year-olds were up-to-date with screening at recommended intervals: colonoscopy in the last 10 years (26\%), or fecal occult blood test (FOBT) in the last 2 years (7\%), or both (7\%). (Braun et al. Under review; Fedewa et al. 2015)

While European guidelines recommend organized screening programs for CRC, many countries, including Switzerland, have not yet implemented programs at a national level. (Schreuders et al. 2015) In July 2013 universal basic health insurance started reimbursing CRC screening with FOBT and colonoscopy for those aged 50-69 in Switzerland. In 2015, the first organized, state wide, screening program in Switzerland began first in the canton of Vaud and expanded to Uri; the programs target residents between the ages of 50 and 69 and participants have their insurance deductible waived. Over the next few years, the target population $\left(\mathrm{N}=170^{\prime} 000\right)$ of the canton of Vaud will be sent individual invitations with a decision aid for CRC screening, and invited to discuss CRC screening with their family physician. (Auer et al. 2015) The Vaud program offers each eligible citizen the chance to (a) make an informed choice with their family physician to screen for CRC; and (b) to choose between FOBT and colonoscopy. CRC screening is opportunistic and mostly initiated in primary care for most other Swiss citizens, comparable to the U.S.

One of the most advanced FOBTs is the fecal immunochemical test (FIT), also called immunological FOBT (iFOBT). FIT can detect CRC at a rate similar to colonoscopy, but they cannot detect as many polyps or advanced polyps as colonoscopies. (Salas et al. 2014) Recent modelling from CISNET suggests that the absolute number of lifetime CRC deaths averted by a yearly FIT is 23 per 1000 individuals screened; colonoscopy once a decade averts 25 per 1000 CRC deaths (Knudsen et al. 2016), but it is an invasive procedure with rare but serious adverse effects. Patients must take a time off work for a colonoscopy, prepare their bowels (an unpleasant process for 
most), and endure the exam itself. Patients who choose FIT can avoid these requirements, but must sample their own stool at home and mail the test to the laboratory. Patients who use FIT must undergo colonoscopy only if blood is detected in the stool sample (6-8\% of samples in average risk populations). In a context where CRC screening is essentially based on screening with colonoscopy, offering the choice of FIT to patients in addition to colonoscopy may increase CRC screening overall.(Inadomi et al. 2012)

There is little international data on variations in care between primary care physicians' (PCP) practices that offer or recommend CRC tests to patients, but evidence from Switzerland and the US suggests most PCPs prescribe only colonoscopy, and few prescribe FOBT. (Selby et al. 2016; Weiss et al. 2013) There may be a mismatch between what patients are offered and what they may prefer. (Hawley et al. 2014) In an RCT in primary care practices in the US, when patients were offered only colonoscopy, only $38 \%$ were screened, but when they were offered only an FOBT, $67 \%$ were screened. When both FOBT and colonoscopy were offered, $69 \%$ were screened, and a roughly equal number of patients opted for each test. (Inadomi et al. 2012) Recent guidelines from the US Services Preventive Task Force (USPSTF) highlighted the difficulty of balancing between risks and benefits of $\mathrm{CRC}$ screening options. Since the options are variably acceptable to patients and because offering FIT in addition to colonoscopy might increase overall screening rates, the guideline suggested PCPs share decision with their patients to elicit their patient's preferences and increase adherence to screening. (USPSTF 2016) The role of "well-supported and unhurried conversations between physicians and patients" about CRC screening options was recently highlighted in a review that discussed ethical issues in the design and implementation of populationbased health programs.(DeCamp et al. 2018)

We set out to determine overall testing rates and method of CRC testing among patients 50-75 years old who visited a representative sample of PCPs participating in the Swiss Sentinel Surveillance Network (Sentinella). We wanted to identify the proportion of tested for CRC within the specified time period (colonoscopy within 10 years, or FOBT within 2 years), and the method with which they had been tested (colonoscopy/FOBT/other), describe variations in CRC testing between physicians, and to determine the physician-level factors associated with those proportions and methods. 


\section{Method}

\section{Study Setting and Data Collection}

The Swiss Sentinel Surveillance (Sentinella) network is a cooperative surveillance project of the Federal Office of Public Health (FOPH). A convenience sample of 150 to 250 general practitioners, internists, and pediatricians in private practices voluntarily report weekly morbidity data to the network. Participating PCPs report irreversibly anonymized patient data collected during consultations. The number of participating Physicians varies from year to year. In 2017 there were 129 Physicians reporting data for Sentinella (pediatricians excluded), who were invited to participate in our data collection. We used a paper data collection form that we developed with PCPs and specialist physicians in public health and epidemiology. We refined the form in a pilot test with 10 PCPs. The design is based on a comprehensible algorithm that limits the amount of data collected to fit the routine and busy schedule of PCPs. To ease and standardize data collection, we gave PCPs examples of completed data collection forms, short summaries, and showed them an 8-minute online instruction video (French and German only) that explained the data collection process. Following the usual process of collecting data in Sentinella, we sent the form to the FOPH, which distributed it to participating primary care practices in April 2017. PCPs were asked to collect data as soon as possible, but could choose when to start. In practices where more than one PCP reported to the Sentinella network, we asked that only one collect data. Completed forms were sent to the FOPH, which transferred them to us, ensuring the data was twice anonymized and de-identified when necessary. Data collection was complete at the end of December 2017.

The ethics committee of the canton of Bern waived ethical approval for the study because of the double irreversible patient-data anonymization process (the FOPH cannot identify the patients, and investigators cannot identify the PCPs), so our study fell outside of the scope of the Swiss Human Research Act (REQ-2017-00280).

Measures, Outcomes and Covariates 
Physicians were asked to systematically include 40 consecutive patients, aged 50-75 years, seen for a non-urgent face-to-face consultation for more than 5 minutes. PCPs had to follow a strict algorithm for reporting patient data (Online Resource 2) on the collection form. It was up to the PCPs to gather the data on previous CRC testing, either by reviewing the medical records, by asking patients directly during the visit, or both. PCPs who felt overburdened with including every consecutive patient who met the inclusion criteria could choose to include only the first two eligible patients per half-day of work, spreading data collection over a longer period of time. This study is concerned with answers to the first section of the form, where PCPs reported patients' age (birth year only), sex (male, female), and previous CRC testing (colonoscopy $<$ or $>10$ years, FOBT $<$ or $>2$ years, other tests, no tests or unknown). The data form only asked PCPs to report if tests had already been performed, and did not ask PCPs to distinguish between diagnostic or screening tests since diagnostic CRC tests can also have a preventive effect, and a test for either reason would qualify as meeting recommendations on testing intervals.(Stock et al. 2011) The data form included no option to code both a colonoscopy and FOBT if patients had had both examinations within the recommended time intervals. The PCP decided which test to prioritize. For previous FOBT testing, we did not ask PCPs to distinguish between guaiac based FOBT (gFOBT) and immunological FOBT (iFOBT, or FIT) on the data collection form. PCPs also reported the number of weeks it took them to collect data on 40 patients. PCPs indicated if patients had already be seen during data collection. If patients had already been seen, we excluded additional visits from our analysis to ensure we did not include the same patient more than once.

The FOPH gave us PCP-level data, including sex, age (30-39, 40-49, 50-59 and >60), language region (German, French, Italian), and practice location defined according to size, population density, and accessibility criteria by the Federal Statistics Office (urban, intermediate, rural). (Federal Statistics Office 2017)

\section{Statistical analyses}

We applied descriptive statistics to present the sample of participating PCPs and the patients they included. We calculated overall proportions of a) patients tested for CRC and b) method by which they were tested. We also calculated the proportion within each PCP practice of a) patients tested 
for CRC within the specified time periods, and b) testing method used. Then we compared proportions between PCPs. We reported the variation between PCPs in range and quartiles of a) proportion of tested patients, and b) proportion of FOBT among all tested patients.

We used two multivariate mixed effects logistic regressions models to measure the association between PCP demographics and a) proportion of patients tested with colonoscopy within 10 years or FOBT within 2 years compared to no testing and b) FOBT within 2 years or not. The models allow for the clustering of data by PCP. We modeled a random effect by PCP. We modeled fixed effects for patient characteristics (age and sex) and PCP characteristics (age, sex, language region, practice location).

PCPs' representativeness was determined by comparing their demographic characteristics to a representative Swiss sample of 200 PCPs, also used to develop the Swiss Primary Care Active Monitoring (SPAM) program (Selby et al. 2015). We analyzed only available data and did not use specific statistical methods to impute missing data.

All analyses were conducted with Stata version 14.2 (StataCorp LP, College Station, TX, USA).

\section{Results}

Of 129 PCP practices reporting for Sentinella on adult patients, 99 (77\%) had a PCP who agreed to collect data. We excluded data from eight PCPs because two reported data on 20 or fewer patients, four did not fill out the data collection form correctly, one did not include patients as prespecified, and one had missing sociodemographic characteristics. We included 91 PCPs (71\%) in our analysis. Most PCPs $(\mathrm{N}=75)$ indicated they consecutively included all patients that met the inclusion criteria; 14 said they included the first two patients per half-day of work, and 2 did not indicate how they included patients. Mean age of PCPs was 54; three out of four PCPs were men. Half worked in urban regions and two-thirds worked in the German-speaking part of Switzerland. Of the 32 PCPs working in French or Italian speaking regions, 15 participated in the organized CRC screening program in Vaud. Compared to a list of Swiss medical associations of 200 PCPs (Selby et al. 2015), participating PCPs tended to come from more urban $(p=0.02)$ practices, and from 
French-Italian language regions $(p=0.02)$. Sociodemographic characteristics of the participating PCPs are summarized in Table 1. 
Table 1 - Sociodemographic characteristics of participating PCP in the 2017 systematic data collection in the Sentinella Network in Switzerland and comparative data from a list of Swiss medical associations of primary care physicians (PCPs) (Selby et al. 2015).

\begin{tabular}{|c|c|c|c|}
\hline & $\begin{array}{l}\text { Participating } \\
\text { PCPs } \\
\mathrm{N}=91 \text { (\%) }\end{array}$ & $\begin{array}{l}\text { Comparison } \\
\text { sample } \\
\mathrm{N}=200 \text { (\%) }\end{array}$ & P value ${ }^{a}$ \\
\hline \multicolumn{4}{|l|}{ Age (years) } \\
\hline $30-39$ & $5(5)$ & & \\
\hline $40-49$ & $16(17)$ & & \\
\hline $50-59$ & $27(29)$ & & \\
\hline$>60$ & $43(47)$ & & \\
\hline Mean age (SD) & $54(10)$ & $53+(7)$ & 0.14 \\
\hline Sex & & & 0.51 \\
\hline Female & $22(24)$ & $42(21)$ & \\
\hline Area & & & 0.02 \\
\hline Urban & $47(52)$ & $70(35)$ & \\
\hline Intermediate & $22(24)$ & $76(38)$ & \\
\hline Rural & $22(24)$ & $54(27)$ & \\
\hline Language & & & 0.02 \\
\hline German & $59(65)$ & $152(76)$ & \\
\hline French & $27(30)$ & $46(23)$ & \\
\hline Italian & $5(5)$ & $2(1)$ & \\
\hline
\end{tabular}

${ }^{\text {a }} \mathrm{P}$-value for mean age generated with two-sample t-test. P-value for sex, age, and language generated with $\mathrm{chi}^{2}$ test.

† Age estimated by adding 25 years to the year when participants received their medical school diploma.

PCP collected data on 3,637 patients. Of these, we excluded 142 patients because they were not 50-75 years old, and 44 because they had already been seen and included during data collection. Age, gender and CRC testing status of the 3,451 included patients are summarized in Table 2 . Mean age of the patients was 63 years, $50 \%$ were male. Half the population was not up-to-date with CRC screening recommendations; $41 \%$ had been tested with colonoscopy within the last 10 years, $4 \%$ with FOBT within the last 2 years, $1 \%$ had had another test and screening status was missing for $4 \%$ of patients (Fig. 1). 
Table 2 - Patient characteristics and colorectal cancer (CRC) testing proportions in the 2017 systematic data collection in the Sentinella Network in Switzerland.

\begin{tabular}{llll}
\hline & $\begin{array}{l}\text { Men } \\
\mathrm{N}=1,709(\%)\end{array}$ & $\begin{array}{l}\text { Women } \\
\mathrm{N}=1,742(\%)\end{array}$ & $\begin{array}{l}\text { Total } \\
\mathrm{N}=3,451(\%)\end{array}$ \\
\hline $\begin{array}{lll}\text { Age (years) } \\
50-59\end{array}$ & & \\
$60-69$ & $639(37)$ & $653(37)$ & $1,292(37)$ \\
$70-75$ & $667(39)$ & $692(40)$ & $1,359(40)$ \\
& $403(24)$ & $397(23)$ & $800(23)$ \\
Mean (SD) & & & \\
& $62.7(7)$ & $62.7(7)$ & $62.7(7)$ \\
CRC testing status (\%) & & & \\
No up-to-date examination & $878(51)$ & $847(49)$ & $1,725(50)$ \\
Colonoscopy<10 years & $672(39)$ & $733(42)$ & $1,405(41)$ \\
FOBT <2 years & $71(4)$ & $80(5)$ & $151(4)$ \\
Other test & $16(1)$ & $12(1)$ & $28(1)$ \\
Unknown & $72(4)$ & $70(4)$ & $142(4)$ \\
\hline
\end{tabular}

Figure 1 - Proportion of patients not up to date with colorectal cancer (CRC) testing, tested for CRC by colonoscopy within the last 10 years, fecal occult blood test (FOBT) within the past 2 years, or other test. Systematic data collection in the Sentinella Network in Switzerland in 2017.

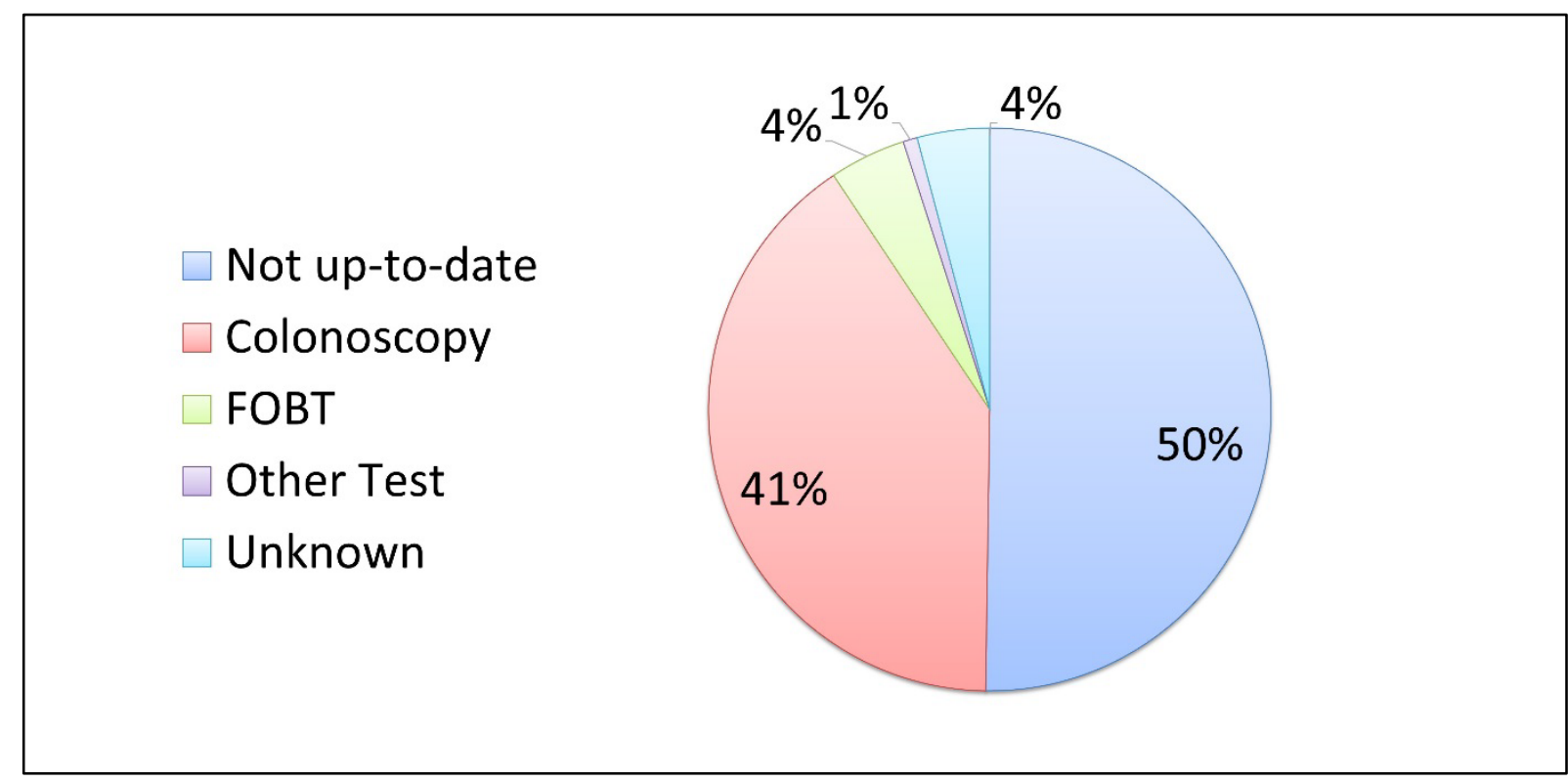

Median proportion of patients tested for CRC within the specified time period per PCP $(\mathrm{N}=91)$ was 45\% (mean 46\%, range 8\%-86\%, IQR: 36\%-57\%). See Fig. 2, Panel A for variation between PCPs in the proportion of patients they tested for CRC. The proportion of patients tested with FOBT among all tested patients per PCP is shown in Fig. 2, Panel B. 54 PCPs (59\%) had no patients tested with FOBT. The median proportion of patients tested with FOBT within 2 years per PCP 
(N=91) was 0\% (Range 0\%-90.9\%, IQR: 0\%-10\%). In multivariate adjusted analyses, older patients were more likely to have been tested for CRC (Table 3). Patients seen by PCPs in the French- or- Italian-speaking part of Switzerland were more likely than those from the Germanspeaking part of Switzerland to have been tested with FOBT then to have had no FOBT testing (Table 4).

Figure 2 - Proportion of patients tested for colorectal cancer (CRC) within each primary care physician (PCP) practice (Panel A) and proportion of patients tested with fecal occult blood test (FOBT) among all tested patients within each PCP practice (Number of PCPs with at least one patient tested with FOBT: 37/91) (Panel B). Systematic data collection in the Sentinella Network in Switzerland in 2017.
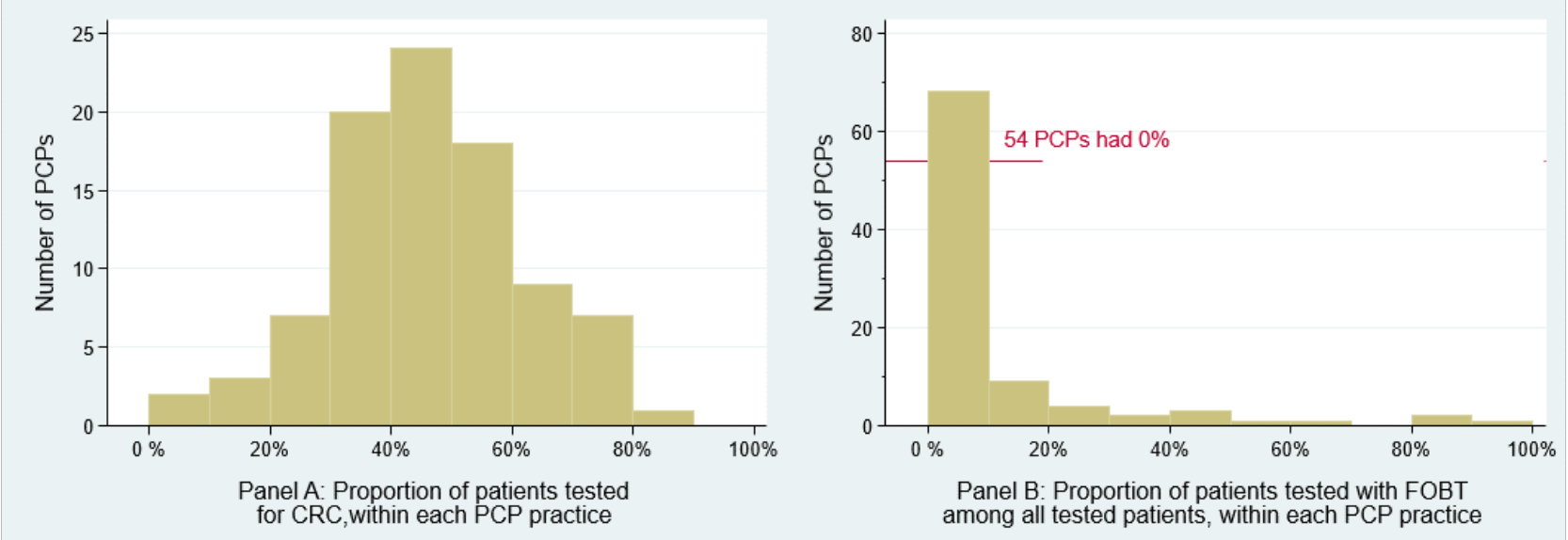
Table 1 - Covariates associated with previous colorectal cancer (CRC) testing of 40 consecutive patients within each primary care physician (PCP) practice that collected data during the 2017 systematic data collection in the Sentinella Network in Switzerland (N PCPs $=91, \mathrm{~N}$ patients=3451) ${ }^{\text {a }}$

OR

Age PCP (ref: $30-39$ years)

$40-49$

$50-59$

$>60$

Sex PCP (ref: male)

Female

Language Region (ref: German)

French / Italian

Practice Location (ref: Urban)

Intermediate

Rural

Age Patients (ref: 50-59 years)

60-69

70-75

1.01

1.05

$\begin{array}{lll}1.42 & 0.66 \text { to } 3.04 & 0.37 \\ 0.95 & 0.47 \text { to } 1.93 & 0.88 \\ 0.92 & 0.47 \text { to } 1.82 & 0.82\end{array}$

0.71 to 1.43

0.96

0.78 to 1.48

0.66

0.69 to 1.58

0.81

0.67

0.41 to 1.11

0.12

$$
1.87
$$

1.58 to 2.20

$<0.01$

2.44

2.01 to 2.95

$<0.01$

Sex Patients (ref: male)

Female

1.08

0.94 to 1.25

0.28

${ }^{a}$ Results from a multivariate mixed effects logistic regression model with previous CRC testing with colonoscopy within the last 10 years or FOBT within the last two years as the outcome, a random effect by PCP and fixed effects for patient-level and PCP-level covariates listed in the table. 
Table 4 - Covariates associated with previous colorectal cancer (CRC) testing with fecal occult blood test (FOBT) of 40 consecutive patients within each primary care physician (PCP) practice that collected data during the 2017 systematic data collection in the Sentinella Network in Switzerland (N PCPs=91, N patients=3451) . $^{\text {. }}$

$\begin{array}{lll}\mathrm{OR} & 95 \% \mathrm{Cl} & \mathrm{P} \text { Value }\end{array}$

\begin{tabular}{|c|c|c|c|}
\hline \multicolumn{4}{|c|}{ Age PCP (ref: $30-39$ years) } \\
\hline $40-49$ & 0.64 & 0.03 to 12.59 & 0.77 \\
\hline $50-59$ & 1.23 & 0.08 to 19.59 & 0.88 \\
\hline$>60$ & 0.92 & 0.29 to 45.76 & 0.32 \\
\hline \multicolumn{4}{|l|}{ Sex PCP (ref: male) } \\
\hline Female & 0.55 & 0.14 to 2.22 & 0.40 \\
\hline \multicolumn{4}{|c|}{ Language Region (ref: German) } \\
\hline French / Italian & 8.06 & 2.38 to 27.35 & $<0.01$ \\
\hline \multicolumn{4}{|c|}{ Practice Location (ref: Urban) } \\
\hline Intermediate & 1.30 & 0.28 to 6.11 & 0.74 \\
\hline Rural & 0.67 & 0.08 to 5.59 & 0.71 \\
\hline \multicolumn{4}{|c|}{ Age Patients (ref: $50-59$ years) } \\
\hline $60-69$ & 1.05 & 0.60 to 1.85 & 0.85 \\
\hline $70-75$ & 1.18 & 0.65 to 2.16 & 0.59 \\
\hline \multicolumn{4}{|c|}{ Sex Patients (ref: male) } \\
\hline Female & 1.45 & 0.93 to 2.27 & 0.1 \\
\hline
\end{tabular}

${ }^{a}$ Results from a multivariate mixed effects logistic regression model with previous CRC testing with FOBT within the last 2 years as the outcome, a random effect by PCP and fixed effects for patient-level and PCP-level covariates listed in the table.

\section{Discussion}

Among patients who visited Swiss primary care practices in $2017,46 \%$ were up-to-date with CRC screening; $89 \%$ of these were tested with colonoscopy. The proportion of patients tested for CRC within the specified time period, and the tests used, varied widely across PCPs. French-or Italianspeaking physicians, half of whom are involved in the Vaud CRC screening program, used FOBT more often than their German-speaking colleagues.

Among participating PCPs in Switzerland, we found 41\% of patients aged 50 to 75 had been tested with colonoscopy in the last 10 years. A 2017 meta-analysis reported on endoscopy (colonoscopy + sigmoidoscopy) rates worldwide; in the US, rates for lifetime colonoscopy ranged from $31 \%$ to $63 \%$ whereas for countries other than the U.S. (Germany, Italy, Spain, the Netherlands, 
Hong Kong, Singapore, South Korea, Switzerland, Canada) rates ranged from $12 \%-44 \%$ for lifetime colonoscopy, and 13-30\% for recent colonoscopy (within 5-10 years) (Chen et al. 2017), except for Germany where, in 2008-2011, 55\% of the screening-eligible population reported colonoscopies within the last 5 to 10 years.

Previous studies reported 33\% of patients were up-to-date with CRC testing in university primary care practices in 2005 and 2006, and 40\% were up-to-date according to the 2012 Swiss Health Survey (Braun et al. Under review; Fedewa et al. 2015; Fischer et al. 2013), but we found higher rates of CRC testing in Switzerland. This difference might be explained by rising rates of CRC testing, especially colonoscopy, in developed countries (Chen et al. 2017); our higher rates may reflect this upward trend. Another explanation is that PCPs volunteer to participate in the Sentinella network, and this self-selected group may be more sensitive to public health issues and more inclined to discuss and offer CRC tests to their patients.

Even though our colonoscopy CRC testing rates exceeded those previously reported, we found much lower rates of FOBT testing (4\%) than were estimated by earlier studies with different designs (13-14\%) (Braun et al. Under review; Fedewa et al. 2015; Fischer et al. 2013). The 2017 meta-analysis did not report information on FOBT but a study in Germany on claims data from 2000-2008 did. They age-standardized their report on the percentage of individuals who had had colonoscopies within $\leq 10$ years and FOBT within the last year and found $23 \%$ of men and $26 \%$ of women were tested with colonoscopy, while and $14 \%$ of men and $22 \%$ of women were tested with FOBT (Stock et al. 2011). A survey of CRC screening programmes worldwide (Klabunde et al. 2015) showed FOBT uptake rates within the last 2 years of $7-68 \%$ and confirms that the rates found in our study are low. We posit three main reasons why our reported FOBT rates were lower than previously reported. First, until recently, guidelines for CRC screening in Switzerland clearly favored colonoscopy and presented FOBT as an inferior option. So we posit that that most PCPs in Switzerland still prefer and mainly offer colonoscopy as a screening option. Second, we report on previous CRC testing, which includes both CRC diagnostics and CRC screening. Guidelines clearly recommend colonoscopy as the preferred to method for ruling out CRC among patients with symptoms of CRC and not FOBT. So, the low proportion of FOBT might reflect the fact that pa- 
tients might have visited their PCP because they had CRC symptoms or other co-morbidities associated with CRC. Previous analyses of the Swiss Health Survey of 2007 and 2012 suggest that those who visited a PCP had a higher colonoscopy/FOBT ratio than those who did not visit a PCP (Braun et al. Under review; Fedewa et al. 2015). Since we report rates of patients who visited their $\mathrm{PCP}$, we expect the proportion of colonoscopy/FOBT to be higher than among the general population. Third, some PCPs may have prioritized colonoscopy over FOBT for people who had had both tests, since only one could be reported in the notification form. This is why the FOBT rates we recorded might not reflect the true rate for those in the Swiss population who did not visit a PCP practice. It also might not reflect the true rate among patients who did visit PCPs since some patients might have had both an FOBT and a colonoscopy, and may only have reported the colonoscopy. This was a weakness of our study design.

Although both tests are recommended for screening, most PCPs had 0/40 patients tested with FOBT and few PCPs had almost all their patients tested with FOBT. If we assume that Swiss patients can choose between the tests at an equal rate, like US patients (Inadomi et al. 2012), then physician preference is likely to be the deciding factor in choice of test rather than patient preference. Higher rates of FOBT testing in French- and Italian-speaking regions of Switzerland (15/32 participating in the CRC screening program in Vaud) might result from the training given to PCPS in the Vaud screening program, since this focuses on offering patients FOBT and colonoscopy on an equal basis.(Selby et al. 2016) Informing patients about different screening options in organized screening programs, teaching PCPs about available tests and urging them to consider patient preferences could help reduce disparities and variation in care.

Strengths of this study include the high adherence of PCPs in the Sentinella network, some of whom have participated in the network for over 30 years. The Network routinely collects nationwide and country representative patient data and has participated in several studies (Gnadinger et al. 2017; Hurlimann et al. 2015; Schmutz et al. 2017), so Sentinella physicians are practiced in providing quality data. The data collection form was developed and tested with PCPs, and was based on a comprehensible algorithm that limited the amount of data PCPs needed to collect, and standardized data collection between PCPs. These factors and the clear instruction for collecting data, likely 
explain the very low rate of incorrectly completed data forms (4/98). Unlike the Swiss Health Survey, we did not rely on telephone interviews. Participating physicians could verify CRC testing status in medical records. If they needed more information, PCPs could query their patients directly about previous CRC testing; patients' self-reported data on colonoscopy usually matches their medical records (Dodou and de Winter 2015).

Our study was limited by the simplicity and anonymity of the data collected. Since only one type of test could be reported, colonoscopy may have been reported more often than FOBT or vice versa in patients who had taken both tests. Reporting preference may have differed between PCPs and may have been related to their characteristics. We could not analyze other sociodemographic factors of patients that could have been associated with CRC testing. Our results only apply to the population that visits PCPs, and not to the whole Swiss population. When we compared study participants to a random sample on a list from the Swiss Medical Association, we found that PCPs in urban areas and those practicing in the French and Italian part of Switzerland were overrepresented. PCPs could not indicate on the data collection form which "other tests" patients might have undergone, and thus could not indicate if they were conducted within the recommended time intervals. Since we did not perform a chart-based validation of the data collected, we are unable to assess the quality of the self-report of the data by PCPs. We urge for careful interpretation of the data, since we cannot rule out the possibility of selection bias of participants by PCPs who might not have strictly followed the consecutive inclusion of patients rule. Even if the participating PCPS had experience in rigorously collecting data as a regular reporting activity for the FOPH within the Sentinella network, we cannot eliminate the possibility of information bias. Patients might have underreported FOBT and some PCPs may have been more likely than others to verify previous screening, introducing bias.

Since diagnostic CRC tests can have a preventive effect,(Stock et al. 2011) the data collection form did not distinguish between tests that had been done for diagnostic or screening purposes, so the rate of patients previously tested for CRC is not limited to patients who were tested for screening purposes. Including all past CRC tests helped us correctly identify the population who had not previously been tested for CRC within recommended intervals, and who were thus eligible for CRC 
screening. The data collection form also did not distinguish between previous guaiac-based FOBT (gFOBT) and immunological FOBT (iFOBT).

Future studies should test the effect of interventions promoting shared decision making among PCPs to reduce variation in care between PCPs and increase variation within PCP practices for screening methods for CRC. We are currently testing a multi-component data-driven training program to promote shared decision making in CRC screening decisions. It is intended to increase the proportion of patients who opt for screening and increase the number of PCP practices where patients are tested for FOBT and colonoscopy after making an informed choice.

Among the PCPs reporting for the Sentinella network in Switzerland, less than half of patients between 50-75 years old were up-to-date with CRC screening. Most PCPs never tested their patients with FOBT; a small number only reported using FOBT. PCP preference may explain the preponderance of colonoscopy tests and the low rate of FOBT and the wide variation in testing rates among PCP practices.

\section{Compliance with Ethical Standards}

Conflict of interest: None of the authors identified a conflict of interest for this manuscript.

Funders: This work was supported by the funds from the Swiss National Scientific Foundations National Research Plan 74 (NFP74. 407440_167519). The funder played no role in the design or conduct of the study, in the collection, management, analysis, or interpretation of data, or in the preparation, review, or approval of the manuscript.

Ethical board: The ethics committee of the canton of Bern waived ethical approval for the study because of the double irreversible patient-data anonymization process (the FOPH cannot identify the patients, and investigators cannot identify the PCPs), so our study fell outside of the scope of the Swiss Human Research Act (REQ-2017-00280). 


\section{References:}

Braun AL, Kaessner A, Syrogiannoul L et al. (Under review) Association between colorectal cancer testing and insurance status: evidence from the Swiss Health Interview Surveys 2007 and 2012.

Brenner H, Stock C, Hoffmeister M (2014) Effect of screening sigmoidoscopy and screening colonoscopy on colorectal cancer incidence and mortality: systematic review and meta-analysis of randomised controlled trials and observational studies BMJ 348:g2467 doi:10.1136/bmj.g2467

Chen C, Lacke E, Stock C, Hoffmeister M, Brenner H (2017) Colonoscopy and sigmoidoscopy use among older adults in different countries: A systematic review Prev Med 103:33-42 doi:10.1016/j.ypmed.2017.07.021

DeCamp M, Pomerantz D, Cotts K et al. (2018) Ethical Issues in the Design and Implementation of Population Health Programs J Gen Intern Med 33:370-375 doi:10.1007/s11606-017-4234-4

Dodou D, de Winter JC (2015) Agreement between self-reported and registered colorectal cancer screening: a meta-analysis Eur J Cancer Care (Engl) 24:286-298 doi:10.1111/ecc.12204

Dominic OG, McGarrity T, Dignan M, Lengerich EJ (2009) American College of Gastroenterology Guidelines for Colorectal Cancer Screening 2008 Am J Gastroenterol 104:2626-2627; author reply 2628-2629 doi:10.1038/ajg.2009.419

Federal Statistics Office (2017) Nachhaltige Entwicklung, regionale und internationale Disparitäten / Statistische Grundlagen und Übersichten.

Fedewa SA, Cullati S, Bouchardy C et al. (2015) Colorectal Cancer Screening in Switzerland: Cross-Sectional Trends (2007-2012) in Socioeconomic Disparities PLoS One 10:e0131205 doi:10.1371/journal.pone.0131205

Fischer R, Collet TH, Zeller A et al. (2013) Obesity and overweight associated with lower rates of colorectal cancer screening in Switzerland Eur J Cancer Prev 22:425-430 doi:10.1097/CEJ.0b013e32835f3b87

Gnadinger M, Conen D, Herzig L, Puhan MA, Staehelin A, Zoller M, Ceschi A (2017) Medication incidents in primary care medicine: a prospective study in the Swiss Sentinel Surveillance Network (Sentinella) BMJ Open 7:e013658 doi:10.1136/bmjopen-2016-013658

Hawley S, Lillie S, Cooper G, Elston Lafata J (2014) Managed care patients' preferences, physician recommendations, and colon cancer screening Am J Manag Care 20:555-561

Hurlimann D, Limacher A, Schabel M et al. (2015) Improvement of antibiotic prescription in outpatient care: a cluster-randomized intervention study using a sentinel surveillance network of physicians $J$ Antimicrob Chemother 70:602-608 doi:10.1093/jac/dku394

Inadomi JM, Vijan S, Janz NK et al. (2012) Adherence to colorectal cancer screening: a randomized clinical trial of competing strategies Arch Intern Med 172:575-582 doi:10.1001/archinternmed.2012.332

Klabunde C, Blom J, Bulliard JL et al. (2015) Participation rates for organized colorectal cancer screening programmes: an international comparison J Med Screen 22:119-126 doi:10.1177/0969141315584694

Knudsen AB, Zauber AG, Rutter CM et al. (2016) Estimation of Benefits, Burden, and Harms of Colorectal Cancer Screening Strategies: Modeling Study for the US Preventive Services Task Force JAMA 315:2595-2609 doi:10.1001/jama.2016.6828

Lauby-Secretan B, Vilahur N, Bianchini F, Guha N, Straif K, International Agency for Research on Cancer Handbook Working G (2018) The IARC Perspective on Colorectal Cancer Screening N Engl J Med doi:10.1056/NEJMsr1714643

McPhee SJ, Richard RJ, Solkowitz SN (1986) Performance of cancer screening in a university general internal medicine practice: comparison with the 1980 American Cancer Society Guidelines Journal of General Internal Medicine 1:275-281

Salas D, Vanaclocha M, Ibanez J et al. (2014) Participation and detection rates by age and sex for colonoscopy versus fecal immunochemical testing in colorectal cancer screening Cancer causes \& control : CCC 25:985-997 doi:10.1007/s10552-014-0398-y

Schmutz C, Bless PJ, Mausezahl D, Jost M, Mausezahl-Feuz M, Swiss Sentinel Surveillance N (2017) Acute gastroenteritis in primary care: a longitudinal study in the Swiss Sentinel Surveillance Network, Sentinella Infection 45:811-824 doi:10.1007/s15010-017-1049-5

Schreuders EH, Ruco A, Rabeneck L, Schoen RE, Sung JJ, Young GP, Kuipers EJ (2015) Colorectal cancer screening: a global overview of existing programmes Gut 64:1637-1649 doi:10.1136/gutjnl-2014309086 
Selby K, Cornuz J, Gachoud D et al. (2016) Training primary care physicians to offer their patients faecal occult blood testing and colonoscopy for colorectal cancer screening on an equal basis: a pilot intervention with before-after and parallel group surveys BMJ Open 6:e011086 doi:10.1136/bmjopen-2016-011086

Selby K, Cornuz J, Senn N (2015) Establishment of a Representative Practice-based Research Network (PBRN) for the Monitoring of Primary Care in Switzerland J Am Board Fam Med 28:673-675 doi:10.3122/jabfm.2015.05.150110

Stock C, Brenner H (2010) Utilization of lower gastrointestinal endoscopy and fecal occult blood test in 11 European countries: evidence from the Survey of Health, Aging and Retirement in Europe (SHARE) Endoscopy 42:546-556 doi:10.1055/s-0029-1244127

Stock C, Ihle P, Schubert I, Brenner H (2011) Colonoscopy and fecal occult blood test use in Germany: results from a large insurance-based cohort Endoscopy 43:771-781 doi:10.1055/s-0030-1256504

USPSTF (2016) Screening for Colorectal Cancer: US Preventive Services Task Force Recommendation Statement JAMA 315:2564-2575 doi:10.1001/jama.2016.5989

von Karsa L, Patnick J, Segnan N et al. (2013) European guidelines for quality assurance in colorectal cancer screening and diagnosis: overview and introduction to the full supplement publication Endoscopy 45:51-59 doi:10.1055/s-0032-1325997

Weiss JM, Smith MA, Pickhardt PJ et al. (2013) Predictors of colorectal cancer screening variation among primary-care providers and clinics Am J Gastroenterol 108:1159-1167 doi:10.1038/ajg.2013.127 
Online Resource 1 - Data collection form used by primary care physicians (PCPs) in the Sentinella network in Switzerland in 2017.

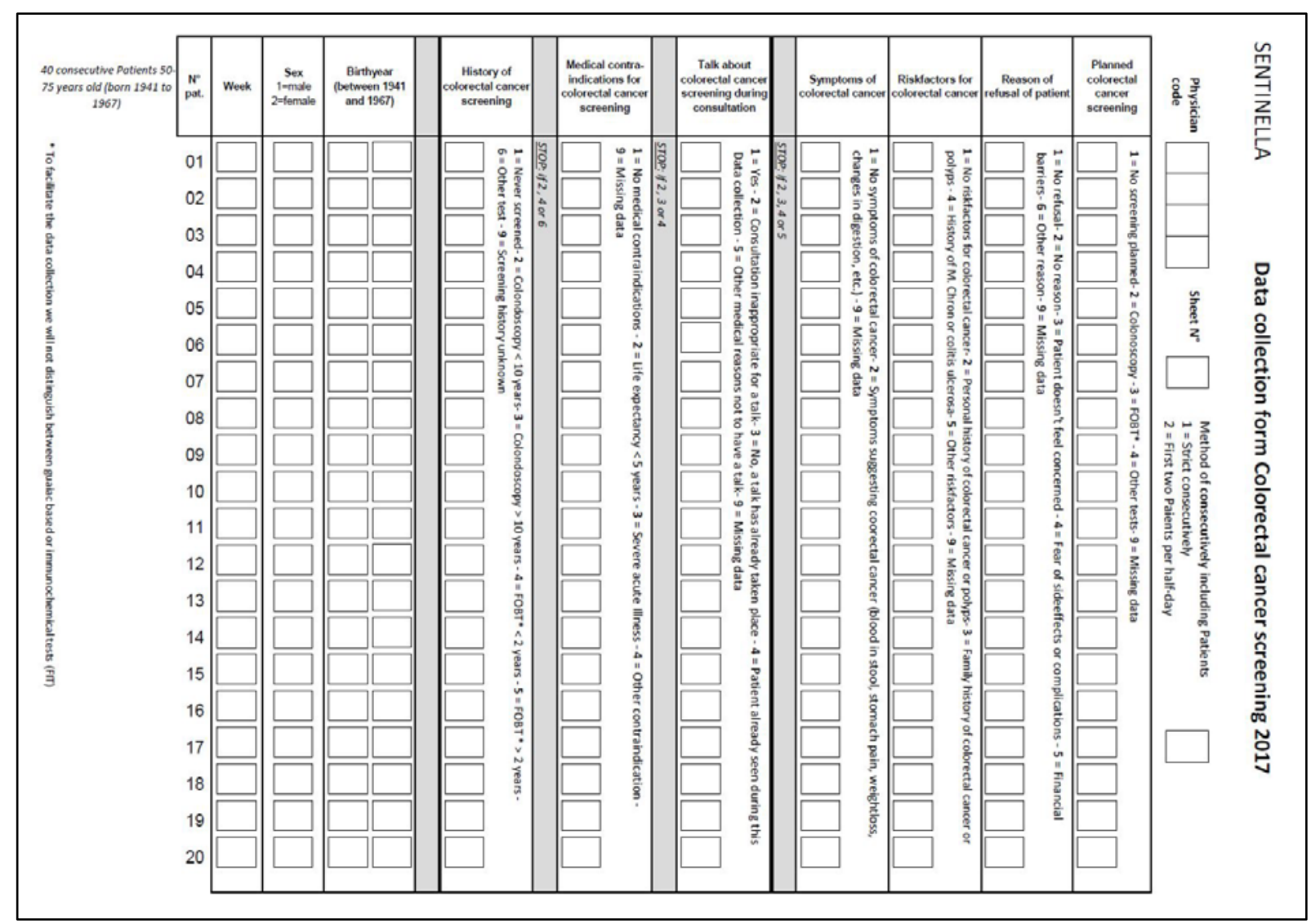


Online Resource 2 - Data collection algorithm used by primary care physicians (PCPs) in the Sentinella network in Switzerland in 2017.

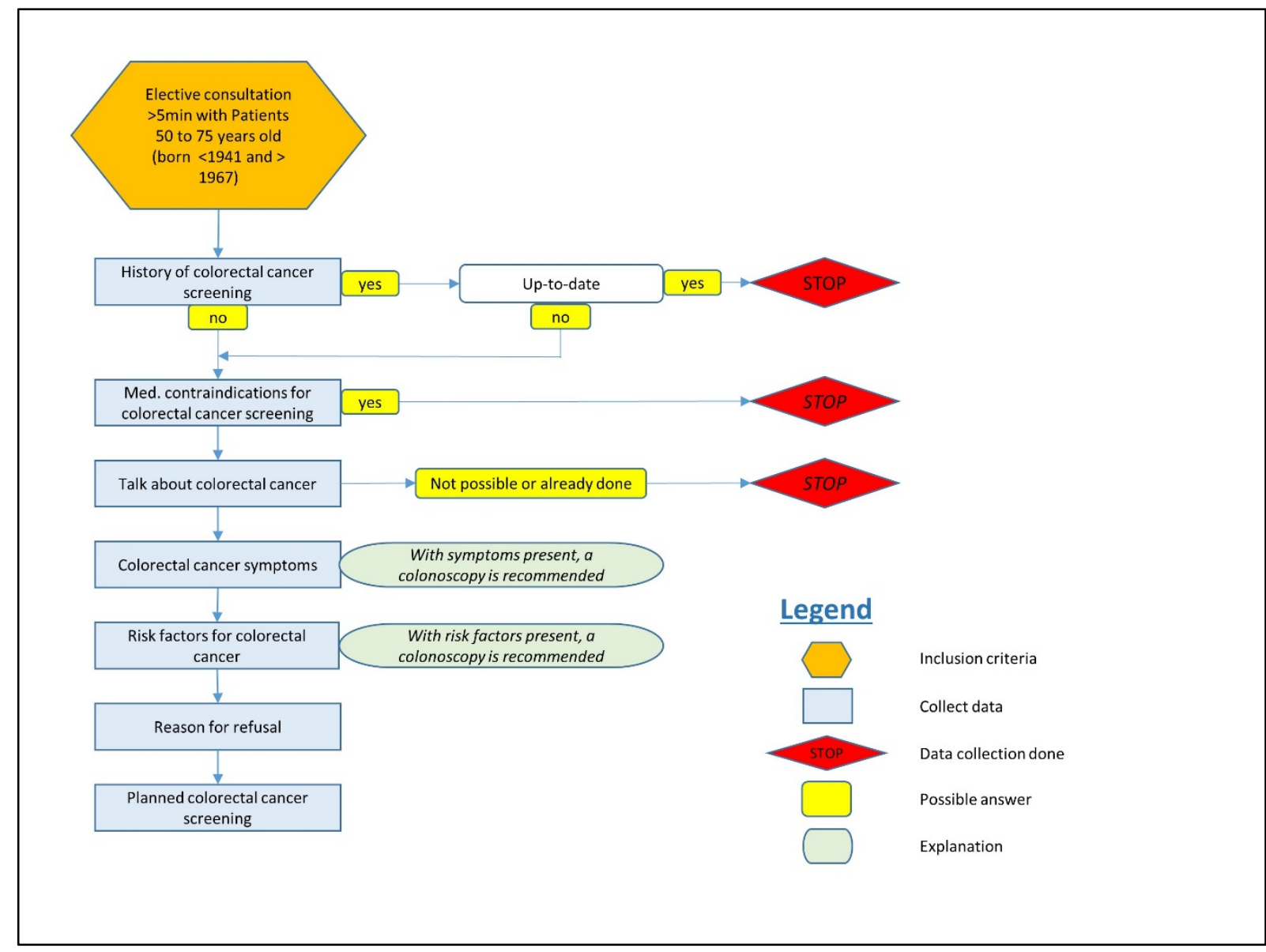

